\title{
Coupled channel model of the scalar isovector meson photopro- duction
}

\author{
Ł. Bibrzycki ${ }^{1, a}$ and R.Kamiński² \\ ${ }^{1}$ Institute of Computer Science, Pedagogical University of Cracow, Podchorążych 2, 30-084 Kraków, Poland \\ ${ }^{2}$ Institute of Nuclear Physics, Polish Academy of Sciences, Radzikowskiego 152, 31-342 Kraków, Poland
}

\begin{abstract}
We present the coupled channel model of the scalar isovector resonance photoproduction including the $\pi \eta, K \bar{K}$ and $\pi \eta^{\prime}$ channels and calculate resulting mass distribution and the cross section in the $\pi \eta$ channel. We show that the shape of this mass distribution, is strongly affected by the phase of background amplitude. We also discuss the effect of inclusion the $\pi \eta^{\prime}$ channel on the overall isovector photoproduction process.
\end{abstract}

\section{Introduction and motivation}

Electromagnetic processes are known to be a good source of information on the meson inner structure. Analysis of these processes turned out to be particularly fruitful in case of scalar mesons which emerge eg. in the $\phi(1020)$ radiative decays in both $\pi \pi$ and $\pi \eta$ channels. Photoproduction of isoscalar and isovector scalar resonances can be treated as a complementary source of information on the scalar meson structure. Here we are concerned with the photoproduction of isovector scalar resonances, particularly the $a_{0}(980)$. Moreover, we are interested in photoproduction at photon energies of about $10 \mathrm{GeV}$, ie. energies achievable in new JLab experiments CLAS12 and GlueX. In this kinematic region the process is dominated by the $\mathrm{t}$ channel meson exchange which leads to production of pseudoscalar pairs $\pi \eta, K \bar{K}$ and $\pi \eta^{\prime}$. These in turn can resonantly interact in the final state as shown in Fig.1. So, construction of the photoproduction amplitudes is inevitably the coupled channel problem. In Ref.[1]

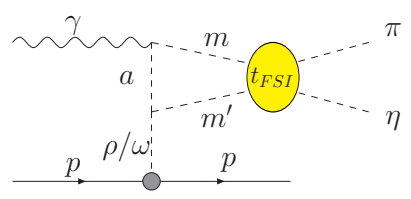

Figure 1. Diagram for the photoproduction process with final state interactions.

we constructed the $\pi \eta-K \bar{K}$ coupled channel photoproduction amplitudes where $a_{0}(980)$ and $a_{0}(1450)$ resonances emerged due to final state interactions. Here we present the extended version of this model which takes into account also the $\pi \eta^{\prime}$ channel. The model is also applicable to higher partial waves

ae-mail: lukasz.bibrzycki@ifj.edu.pl 
(see [2]) and is relevant in the context of CLAS12 and GlueX experiments to be started shortly at Jefferson Laboratory. In these experiments the $\pi \eta, K \bar{K}$ and $\pi \eta^{\prime}$ pairs will be photoproduced copiously (also by polarized photons) enabling the partial wave analysis. Reliable models of the resonance photoproduction are thus timely and opportune.

In practical situations the resonance signal observed in the $\pi \eta, K \bar{K}$ and $\pi \eta^{\prime}$ mass distributions is accompanied by the background resulting from the direct pseudoscalar pair production, Drell mechanism [3] or cascade baryon resonance de-excitation [4]. The baryon resonance contribution to the background is believed to decrease with photon increasing energy but as we show below, its phase strongly affects the shape of mass distribution.

\section{General amplitude structure}

Our model assumes that the scalar resonance photoproduction is a two stage process. First, a pair of pseudoscalar mesons $\pi \eta, K^{0} \overline{K^{0}}, K^{+} K^{-}$or $\pi \eta^{\prime}$ is photoproduced, which we describe in terms of Born amplitudes. The final state rescattering is described in terms of the coupled channel and unitary model. The complete $\pi \eta$ photoproduction amplitude, which takes into account the final state rescattering reads

$$
\left\langle\lambda^{\prime}\left|A_{\pi \eta}\right| \lambda_{\gamma} \lambda\right\rangle=\left\langle\lambda^{\prime}\left|V_{\pi \eta}\right| \lambda_{\gamma} \lambda\right\rangle+4 \pi \sum_{\left\{m m^{\prime}\right\}} \int_{0}^{\infty} \frac{k^{\prime 2} d k^{\prime}}{(2 \pi)^{3}} F\left(k^{\prime}\right) t_{\pi \eta ; m m^{\prime}} G_{m m^{\prime}}\left\langle\lambda^{\prime}\left|V_{m m^{\prime}}\right| \lambda_{\gamma} \lambda\right\rangle
$$

where $V_{\pi \eta}\left(V_{m m^{\prime}}\right)$ is the Born amplitude of the $\pi \eta\left(\mathrm{mm}^{\prime}\right)$ pair photoproduction, $t_{\pi \eta ; m m^{\prime}}$ is the coupled channel rescattering amplitude, $\lambda, \lambda^{\prime}$ and $\lambda_{\gamma}$ are respectively the helicities of the initial and final proton, and photon helicity. $G_{m m^{\prime}}$ is the propagator of the intermediate $\mathrm{mm}^{\prime}$ pair defined as

$$
G_{m m^{\prime}}=\frac{-1}{M_{m m^{\prime}}-M_{m m^{\prime}}^{\prime}\left(k^{\prime}\right)+i \epsilon}
$$

and $F\left(k^{\prime}\right)$ is the form-factor needed to regularize the divergent mesonic loop of the diagram shown in Fig. 1. Projecting out the higher partial waves, we arrive at the following coupled channel $S$-wave $\pi \eta$ photoproduction amplitude:

$$
A_{\pi \eta}=\left[1+i r_{\pi \eta} t_{\pi \eta}^{I=1}\right] V_{\pi \eta}+i r_{K \bar{K}} t_{\pi \eta ; K}^{I=1} \frac{1}{\sqrt{2}}\left(V_{K^{+} K^{-}}+V_{K^{0}} \overline{K^{0}}\right)+i r_{\pi \eta^{\prime}} t_{\pi \eta ; \pi \eta},
$$

where $r_{\pi \eta}=-k_{\pi \eta} M_{\pi \eta} / 8 \pi, r_{K \bar{K}}=-k_{K} M_{\pi \eta} / 8 \pi, r_{\pi \eta^{\prime}}=-k_{\pi \eta^{\prime}} M_{\pi \eta} / 8 \pi$ and $M_{\pi \eta}$ is effective mass of the $\pi \eta$ system. The $t_{\pi \eta}^{I=1}, t_{K \bar{K} ; \pi \eta}^{I=1}$ and $t_{\pi \eta^{\prime} ; \pi \eta}^{I=1}$ are isovector elastic $\pi \eta$ amplitude and transition amplitudes respectively. The $k_{\pi \eta}, k_{K \bar{K}}$ and $k_{\pi \eta^{\prime}}$ are $\mathrm{CM}$ momenta in the $\pi \eta, K \bar{K}$ and $\pi \eta^{\prime}$ channels. In this introductory study we neglect the effects of the off-shell propagation of intermediate mesons. Thus, the third term in Eq.(3) vanishes below the $\pi \eta^{\prime}$ threshold and does not affect the amplitude for masses corresponding to $a_{0}(980)$ resonance. Further we use numerical channel indices for the elements of the final state scattering amplitude and denote $t_{\pi \eta}$ as $T_{11}, t_{\pi \eta ; K \bar{K}}$ as $T_{12}$ and $t_{\pi \eta ; \pi \eta^{\prime}}$ as $T_{13}$ respectively.

\section{Born amplitudes}

In Fig. 2 we show the general structure of Feynman diagrams representing Born amplitudes. In these diagrams $a$ corresponds to $\rho$ or $\omega$ in the $\pi \eta$ and $\pi \eta^{\prime}$ channels and $K$ or $K^{*}$ in the $K \bar{K}$ channel. $b$ corresponds to the $\rho$ and $\omega$ exchange. The diagram $c$ ) is required by the gauge invariance of charged kaon pair production amplitudes. The general form of amplitudes corresponding to diagrams of Fig. 


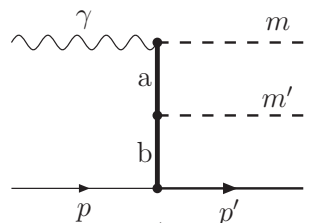

a)

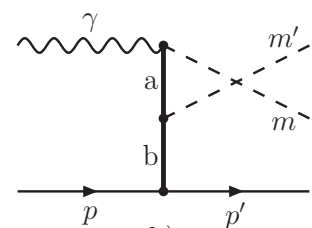

b)

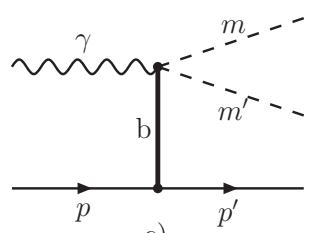

c)

Figure 2. Diagrammatic representation of Born amplitudes for $\pi \eta, K^{0} \overline{K^{0}} K^{+} K^{-}$and $\pi \eta^{\prime}$ intermediate states.

2 reads:

$$
V_{m m^{\prime}}=\sum_{\left\{m m^{\prime}\right\}} \bar{u}\left(p^{\prime}, s^{\prime}\right) J_{r, m m^{\prime}} \cdot \varepsilon\left(q, \lambda^{\gamma}\right) u(p, s),
$$

where the sum is over intermediate states $m m^{\prime}, J_{r, m m^{\prime}}$ is the hadronic current, $u(p, s)$ and $\bar{u}\left(p^{\prime}, s^{\prime}\right)$ wave functions of the initial and final proton respectively and $\varepsilon$ - the polarisation 4-vector of the incident photon. To perform the partial wave expansion of the Born amplitudes we have used procedures discussed in $[1,2]$.

\section{Final state interaction amplitudes}

To obtain the final state rescattering amplitudes one solves the coupled channel Lippman-Schwinger equations which in momentum space read [5-7]

$$
\langle q|\hat{T}| k\rangle=\langle q|\hat{V}| k\rangle+\int \frac{d^{3} p}{(2 \pi)^{3}}\langle q|\hat{V}| p\rangle\langle p|\hat{G}| p\rangle\langle p|\hat{T}| k\rangle,
$$

where $\hat{V}, \hat{G}$ and $\hat{T}$ are $3 \times 3$ matrices in channel space. $\hat{G}$ denotes the diagonal propagator matrix, with the elements defined as

$$
G_{i}(p)=\frac{1}{E-E_{i}(p)+i \epsilon} .
$$

$\hat{V}$ is the interaction matrix which is assumed in a separable form

$$
\left\langle q\left|V_{i j}\right| k\right\rangle=\lambda_{i j} g_{i}(q) g_{j}(k),
$$

where $\lambda_{i j}$ is a $3 \times 3$ coupling constant matrix and the form factor is defined as

$$
g_{i}(p)=\sqrt{\frac{4 \pi}{m_{i}}} \frac{1}{p^{2}+\beta_{i}^{2}},
$$

with $m_{i}$ being the reduced mass of mesons in $i$-th channel. Given the symmetric matrix of couplings and 3 form-factor parameters $\beta_{i}$, the total number of model parameters for 3 channels is 9 . The on-shell scattering matrix elements $T_{i j}\left(k_{i}, k_{j}\right)$ can be expressed in terms of the $S$-matrix elements

$$
S_{i j}=\delta_{i j}-\frac{i}{\pi} \sqrt{k_{i} \alpha_{i} k_{j} \alpha_{j}} T_{i j}\left(k_{i}, k_{j}\right)
$$

where $i$ and $j$ enumerate channels, $k_{i}, k_{j}$ are center of mass momenta in respective channels and $\alpha_{i}$ are defined as:

$$
\alpha_{1}=\frac{E_{\pi} E_{\eta}}{E_{\pi}+E_{\eta}}, \quad \alpha_{2}=\frac{E_{K}}{2}, \quad \alpha_{3}=\frac{E_{\pi} E_{\eta^{\prime}}}{E_{\pi}+E_{\eta^{\prime}}} .
$$


Here $E_{\pi}, E_{\eta}, E_{K}$ and $E_{\eta^{\prime}}$ denote the $\pi, \eta K$ and $\eta^{\prime}$ energies in the center of mass of respective channels. For the 3-channel case, the relation between elements of the $S$-matrix and the Jost function can be described by the following expressions [6]:

$$
S_{11}=\frac{D\left(-k_{1}, k_{2}, k_{3}\right)}{D\left(k_{1}, k_{2}, k_{3}\right)}, \quad S_{22}=\frac{D\left(k_{1},-k_{2}, k_{3}\right)}{D\left(k_{1}, k_{2}, k_{3}\right)}, \quad S_{13}^{2}=S_{11} S_{33}-\frac{D\left(-k_{1}, k_{2},-k_{3}\right)}{D\left(k_{1}, k_{2}, k_{3}\right)} .
$$

Further matrix elements can be obtained by proper change of signs in channel momenta. The experimental data on pseudoscalar meson scattering in the isovector channel is very scarce. So, to evaluate the model parameters we combine the information on the location of resonance poles in the complex momenta planes with the values of known $a_{0}(980)$ branching fractions and amplitude threshold parameters. This approach is the extension of the method used in [7]. One must be aware, however, that the quantities used to constrain model parameters are burdened with large uncertainties which impacts the accuracy of model predictions. The resonance poles of $a_{0}(980)$ and $a_{0}(1450)$ correspond to zeroes of Jost function

$$
D\left(k_{1}^{r}, k_{2}^{r}, k_{3}^{r}\right)=0, \quad D\left(k_{1}^{R}, k_{2}^{R}, k_{3}^{R}\right)=0,
$$

where superscripts $r$ and $R$ relate to $a_{0}(980)$ and $a_{0}(1450)$ respectively. Thus, Eqs.(12) impose 4 constraints on model parameters, see [8] for details of parameter evaluation.

\section{Cross sections and mass distributions}

The double differential cross section for the photoproduction of proton and two pseudoscalars reads

$$
\frac{d \sigma}{d t d M_{\pi \eta}}=\frac{1}{4} \frac{1}{(2 \pi)^{3}} \frac{|k|}{32 m^{2} E_{\gamma}^{2}} \sum_{\lambda_{\gamma}, \lambda, \lambda^{\prime}}\left|\left\langle\lambda^{\prime}\left|A_{\pi \eta}\right| \lambda_{\gamma} \lambda\right\rangle\right|^{2},
$$

where $m$ and $E_{\gamma}$ are the proton mass and photon energy in the laboratory frame respectively. To

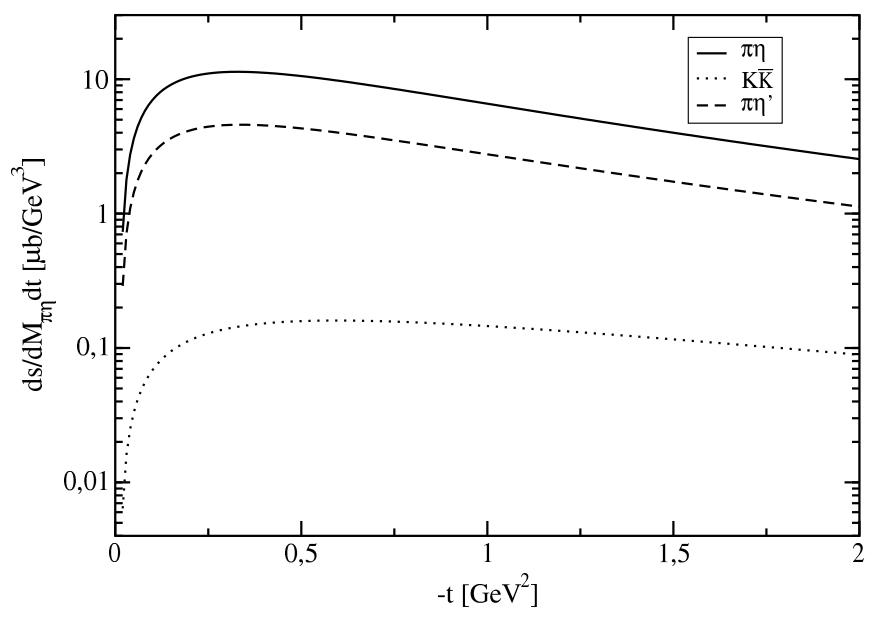

Figure 3. Born cross sections $d \sigma / d M_{\pi \eta} d t$ for the $\pi \eta, K \bar{K}$ and $\pi \eta^{\prime}$ channels at $E_{\gamma}=9 \mathrm{GeV}$ and $M_{\pi \eta}=1.4 \mathrm{GeV}$.

evaluate the relative contribution of $\pi \eta, K \bar{K}$ and $\pi \eta^{\prime}$ channels to the overall process we first calculated the respective $t$ dependent cross sections and mass distributions. Fig. 3 shows that for effective 


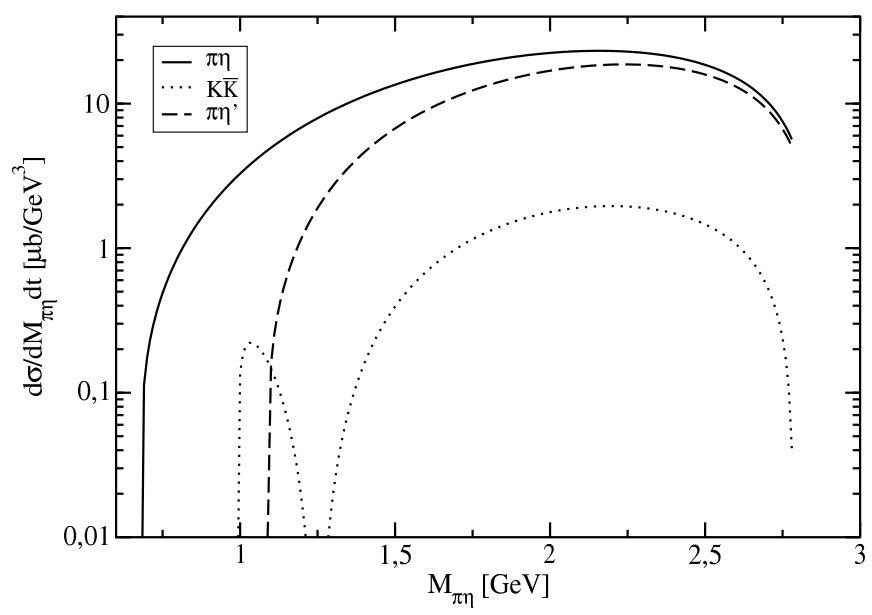

Figure 4. Born mass distributions for $\pi \eta, K \bar{k}$ and $\pi \eta^{\prime}$ channels at $E_{\gamma}=9 \mathrm{GeV}$ and $-t=0.55 \mathrm{GeV}^{2}$

masses corresponding to $a_{0}(1450)$ resonance, the $\pi \eta^{\prime}$ contribution is much stronger that the $K \bar{K}$ contribution. The mass dependence of Born cross section in Fig.4 confirms that the $\pi \eta^{\prime}$ channel may strongly influence the amplitude for masses corresponding to $a_{0}(1450)$. In Fig.5 we show the effects

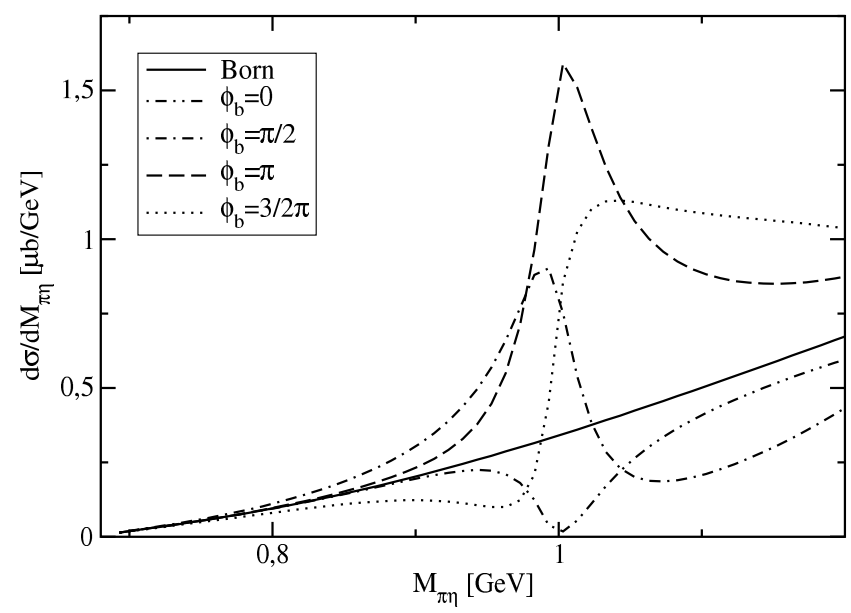

Figure 5. Dependence of the mass distribution on the phase of the background amplitude.

of the real Born amplitude interference with the final state interaction amplitude and conclude that, for real background the resonance is represented by the minimum (double dot-dash line). The resonance is represented by the maximum in the mass distribution only when the background acquires some nonvanishing imaginary part. Dot-dashed, dashed and dotted curves in Fig. 5 represent mass distributions resulting from interference of the final state interactions terms with the background amplitude "corrected" by additional phases of $\frac{\pi}{2}, \pi$ and $\frac{3}{2} \pi$ respectively. 


\section{Summary}

Our introductory study of the three coupled channel scalar isovector photoproduction suggests that the $\pi \eta^{\prime}$ channel gives important contribution to the photoproduction cross section for effective masses above the $\pi \eta^{\prime}$ threshold. For the purely real background resulting from the Born $\pi \eta$ photoproduction amplitudes, the $a_{0}(980)$ resonance is represented by the minimum rather than maximum. To develop the maximum in the mass distribution, the background amplitude must acquire some non-vanishing imaginary part. This imaginary part may arise, for example, from the cascade decays of baryon resonances.

\section{Acknowledgments}

This research has been funded by the Polish National Science Center (NCN) grant No. DEC2013/09/B/ST2/04382

\section{References}

[1] Ł. Bibrzycki, R. Kamiński, Int. J. Mod. Phys. A 31, 1650139 (2016)

[2] Ł. Bibrzycki, R. Kamiński, Phys. Rev. D 87, 114010 (2013)

[3] J. Pumplin, Phys. Rev. D 2, 1859 (1970)

[4] M. Doring, E. Oset and D. Strottman, Phys. Rev. C 73, 045209 (2006)

[5] R. Kamiński, L. Leśniak and J. P. Maillet, Phys. Rev. D 50, 3145 (1994)

[6] L. Leśniak, Acta Phys. Polon. B 27, 1835 (1996)

[7] A. Furman and L. Leśniak, Phys. Lett. B 538, 266 (2002)

[8] R. Kamiński, these proceedings 